\title{
Gene expression profiling analysis of osteosarcoma cell lines
}

\author{
LU SUN ${ }^{*}, \mathrm{JIE} \mathrm{LI}^{*}$ and BING YAN \\ Department of Orthopedics, Shandong Chinese Medical Hospital, Jinan, Shandong 250014, P.R. China
}

Received September 1, 2014; Accepted May 13, 2015

DOI: $10.3892 / \mathrm{mmr} .2015 .3958$

\begin{abstract}
Osteosarcoma (OS) is the most common type of primary bone malignancy and has a poor prognosis. To investigate the mechanisms of osteosarcoma, the present analyzed the GSE28424 microarray. GSE28424 was downloaded from the Gene Expression Omnibus, and included a collective of 19 OS cell lines and four normal bone cell lines, which were used as controls. Subsequently, the differentially expressed genes (DEGs) were screened using the Limma package in Bioconductor. Gene Ontology (GO) and pathway enrichment analysis of the DEGs was performed using the Database for Annotation, Visualization and Integrated Discovery, interactions between the proteins encoded by the DEGs were identified using STRING, and the protein-protein interaction (PPI) network was visualized using Cytoscape. In addition, modular analysis of the PPI network was performed using the Clique Percolation Method (CPM) in CFinder. A total of 1,170 DEGs were screened, including 530 upreguated and 640 downregulated genes. The enriched functions included organelle fission, immune response and response to wounding. In addition, RPL8 was observed to be involved with the ribosomal pathway in module A of the PPI network of the DEGs. PLCG1, SYK and PLCG2 were also involved in the B-cell receptor signaling pathway in module $\mathrm{B}$ and the Fc-epsilon RI signaling pathway in module C. In addition, AURKA (degree=39), MAD2L1 (degree=38), CDCA8 $($ degree $=38)$, BUB1 $($ degree=37) and MELK $($ degree=37) exhibited higher degrees of connectivity in module $\mathrm{F}$. The results of the present study suggested that the RPL8, PLCG1, PLCG2, SYK, MAD2L1, AURKA, CDCA8, BUB1 and MELK genes may be involved in OS.
\end{abstract}

Correspondence to: Dr Jie Li, Department of Orthopedics, Shandong Chinese Medical Hospital, 16369 Jingshi Road, Jinan, Shandong 250014, P.R. China

E-mail: jielijjl1@163.com

${ }^{*}$ Contributed equally

Key words: osteosarcoma, protein-protein interaction network, differentially expressed genes, module analysis

\section{Introduction}

Osteosarcoma (OS) is the most common type of primary bone malignancy derived from primitive mesenchymal cells (1). OS originates predominantly from bone and rarely from soft tissue (2). With a high degree of malignancy, rapid progression and poor prognosis, OS occurs predominantly in teenagers, and, in adolescents between the ages of 15 and 19 years, OS comprises $15 \%$ of all types of solid extra cranial cancer $(3,4)$.

Several studies have been performed to investigate the molecular mechanisms underlying OS. For example, increased expression levels of vascular endothelial growth factor A (VEGFA) have been reported to induce metastasis and OS development (5-7), the major angiogenic factors, VEGF165 and VEGF189, may be critical for neovascularisation in OS (8), and pigment epithelium-derived factor (PEDF) not only induces apoptotic cell death in OS cells, but also suppress the expression of VEGF, resulting in the inhibition of tumor angiogenesis (9). Alterations in p53 have also been found in uncultured OS samples, with a frequency ranging between 18 and $42 \%$, indicating that p53 may be involved in the pathogenesis of OS $(10,11)$. In the context of bone morphogenetic protein (BMP)/small mothers of decapentaplegic (SMAD) signaling, runt-related transcription factor 2 (Runx2) can act as an inducer of apoptosis and suppressor of growth in OS and normal osteoblasts (12). There exists an important role for human epidermal growth factor receptor 2 (HER2) in the promotion of metastatic potential in OS and in aggressive tumor growth (13).

In 2012, Namløs et al (14) used an integrative microarray approach to analyze genome-wide mRNA expression patterns between a panel of 19 EuroBoNeT OS cell lines and four normal bone cell lines, according to the cut-off point of false discovery rate (FDR) <0.05, and obtained 8,982 mRNA probes, which were significantly differently expressed between the two groups. Using the same data used by Namløs et al (14), the present study aimed to further screen for differentially expressed genes (DEGs) using a more strict threshold of FDR $<0.01$ and $\mid \log 2$ fold-change $(F C) \mid>1$, and to analyze the potential functions of the DEGs using Gene Ontology (GO) functional analysis and Kyoto Encyclopedia of Genes and Genomes (KEGG) pathway enrichment analysis. In addition, the present study aimed to construct a protein-protein interaction (PPI) network of these DEGs and screen the modules of the PPI network to identify the interactions/association between the DEGs. 


\section{Materials and methods}

Microarray data. The expression profile of GSE28424, deposited by Namløs et al (14) was downloaded from the Gene Expression Omnibus (GEO; http://www.ncbi.nlm.nih. gov/geo/), which was based on the platform of the GPL13376 Illumina HumanWG-6 v2.0 expression beadchip (Illumina, San Diego, CA, USA). The GSE28424 dataset included a collective of 19 OS cell lines and four normal bone cell lines, of which the latter were used as controls.

DEGs screening. Following downloading of the GSE28424 data, the microarray data was normalized to a linear model of box plots of $\log 2$ [(perfect match probe value)-MM (mismatch probe value)] using quantile normalization (15). Subsequently, the DEGs between the OS patients and normal control cell lines were analyzed using the linear models for microarray data (Limma) package in Bioconductor (http://www.bioconductor.org/packages/release /bioc/html/limma.html) (16). The adjusted P-value (FDR) $<0.01$ and $\mid \log 2 \mathrm{FCl}>1$ were used as the cut-off criteria.

Functional analysis and pathway enrichment analysis. As an integrated and high-throughput data-mining environment, the Database for Annotation, Visualization and Integrated Discovery (DAVID; http://david.abcc.ncifcrf.gov/) analyzes gene lists from high-throughput genomic experiments (17). The aim of Gene Ontology (GO; http://www.geneontology. org/) is to provide access to extensive documentation and perform functional analyses (18) KEGG (http://www.genome. $\mathrm{ad} . j \mathrm{p} / \mathrm{kegg} /$ ) is a comprehensive database resource, which consists of chemical information, genomic information and systems information (19). Using the DAVID online tool, GO functional analysis and KEGG pathway enrichment analysis were performed for the DEGs. In addition, the GO functional enrichment analyses was focussed predominantly on biological process (BP). $\mathrm{P}<0.05$ was used as the cut-off criterion.

PPI network and module construction. The Search Tool for the Retrieval of Interacting Genes (STRING) online software (http://string-db.org/) (20) was used to identify interactions/associations between the proteins encoded by the DEGs, and a combined score $>0.7$ was used as the cut-off criterion. Subsequently, Cytoscape software (http://cytoscape. org/download_old_versions.html) (21) was used to visualize the PPI network and the Clique Percolation Method (CPM) was used in CFinder software (http://cfinder.org/) (22) to screen the modules of the PPI network. The clique value $(\mathrm{k})$ was set to six.

\section{Results}

DEG analysis. A total of 1,170 DEGs were screened, which included 530 upregulated genes and 640 downregulated genes. There were a higher number of downregulated genes, compared with upregulated genes.

Functional analysis and pathway enrichment analysis. The top 10 most enriched GO functions for the DEGs are listed in Table I. For the upregulated genes, the enriched functions in the BP category included organelle fission $(\mathrm{P}=1.19 \mathrm{E}-09)$, cell cycle process $(\mathrm{P}=3.90 \mathrm{E}-09)$ and nuclear division $(\mathrm{P}=8.03 \mathrm{E}-09)$. For the downregulated genes, the enriched functions in the BP category included immune response $(\mathrm{P}=5.15 \mathrm{E}-32)$, defense response $(\mathrm{P}=2.69 \mathrm{E}-28)$ and response to wounding $(\mathrm{P}=1.40 \mathrm{E}-15)$.

The top 10 enriched KEGG pathways for the DEGs are listed in Table II. For the upregulated genes, the enriched pathways included steroid biosynthesis $(\mathrm{P}=0.0023)$, ribosome $(\mathrm{P}=0.0294)$ and aminoacyl-tRNA biosynthesis $(\mathrm{P}=0.0125)$. For the downregulated genes, the enriched pathways included cell adhesion molecules (CAMs, $\mathrm{P}=3.74 \mathrm{E}-05)$, hematopoietic cell lineage $(\mathrm{P}=1.05 \mathrm{E}-04)$ and natural killer cell-mediated cytotoxicity $(\mathrm{P}=1.36 \mathrm{E}-04)$.

PPI network and module construction. The PPI network of the DEGs was revealed to have 590 nodes and 1,888 interactions (Fig. 1). A total of 10 modules were obtained from the PPI network, of which the top six modules (modules A-F) are shown in Fig. 2. The enriched pathways for the DEGs in the six modules are listed in Table III. Module A had 12 nodes and 50 interactions. In this module, all the DEGs were upregulated genes. The enriched KEGG pathways for DEGs in module A included ribosome $(\mathrm{P}=2.65 \mathrm{E}-12)$, which involved ribosomal protein L8 (RPL8).

Module B had six nodes and 15 interactions. In this module, the number of upregulated genes was considerably lower than the number of downregulated genes. For the DEGs in module $\mathrm{B}$, the enriched KEGG pathways included the $\mathrm{B}$-cell receptor signaling pathway $(\mathrm{P}=2.16 \mathrm{E}-07), \mathrm{Fc}$ epsilon RI signaling pathway $(\mathrm{P}=0.0023)$ and natural killer cell-mediated cytotoxicity $(\mathrm{P}=0.0064)$. In module $\mathrm{B}$, the B-cell receptor signaling pathway involved phospholipase $\mathrm{C} \gamma 1$ (PLC $\gamma 1)$, spleen tyrosine kinase (SYK) and phospholipase $\mathrm{C} \gamma 2$ (PLC $\gamma 2$ ).

Module $\mathrm{C}$ had seven nodes and 20 interactions. In this module, the number of upregulated genes was markedly lower than the number of downregulated genes. The enriched KEGG pathways for the DEGs in module $\mathrm{C}$ included the Fc epsilon RI signaling pathway $(\mathrm{P}=4.43 \mathrm{E}-09), \mathrm{Fc} \gamma \mathrm{R}$-mediated phagocytosis $(\mathrm{P}=0.0049)$ and leukocyte transendothelial migration $(\mathrm{P}=0.0075)$. In module $\mathrm{C}$, the Fc-epsilon RI signaling pathway also involved PLC $\gamma 1$, SYK and PLC $\gamma 2$.

Module D had seven nodes and 20 interactions. In this module, the number of upregulated genes was higher than the number of downregulated genes. For the DEGs in module D, the enriched KEGG pathways included purine metabolism $(\mathrm{P}=2.31 \mathrm{E}-08)$, pyrimidine metabolism $(\mathrm{P}=6.15 \mathrm{E}-05)$ and the cytosolic DNA-sensing pathway $(\mathrm{P}=0.0529)$. The purine metabolism pathway involved adenylate cyclase 2 (ADCY2), NME/NM23 nucleoside diphosphate kinase 1 (NME1) and pyruvate kinase, liver and red blood cell (PKLR).

Module E had 15 nodes and 102 interactions. In this module, the number of upregulated genes was significantly lower than the number of downregulated genes. The enriched KEGG pathways for DEGs in module E included neuroactive ligand-receptor interaction ( $\mathrm{P}=1.10 \mathrm{E}-05)$, chemokine signaling pathway $(\mathrm{P}=6.95 \mathrm{E}-04)$ and cytokine-cytokine receptor interaction $(\mathrm{P}=0.0210)$. The neuroactive ligand-receptor interaction pathway involved complement component 5a receptor 1 


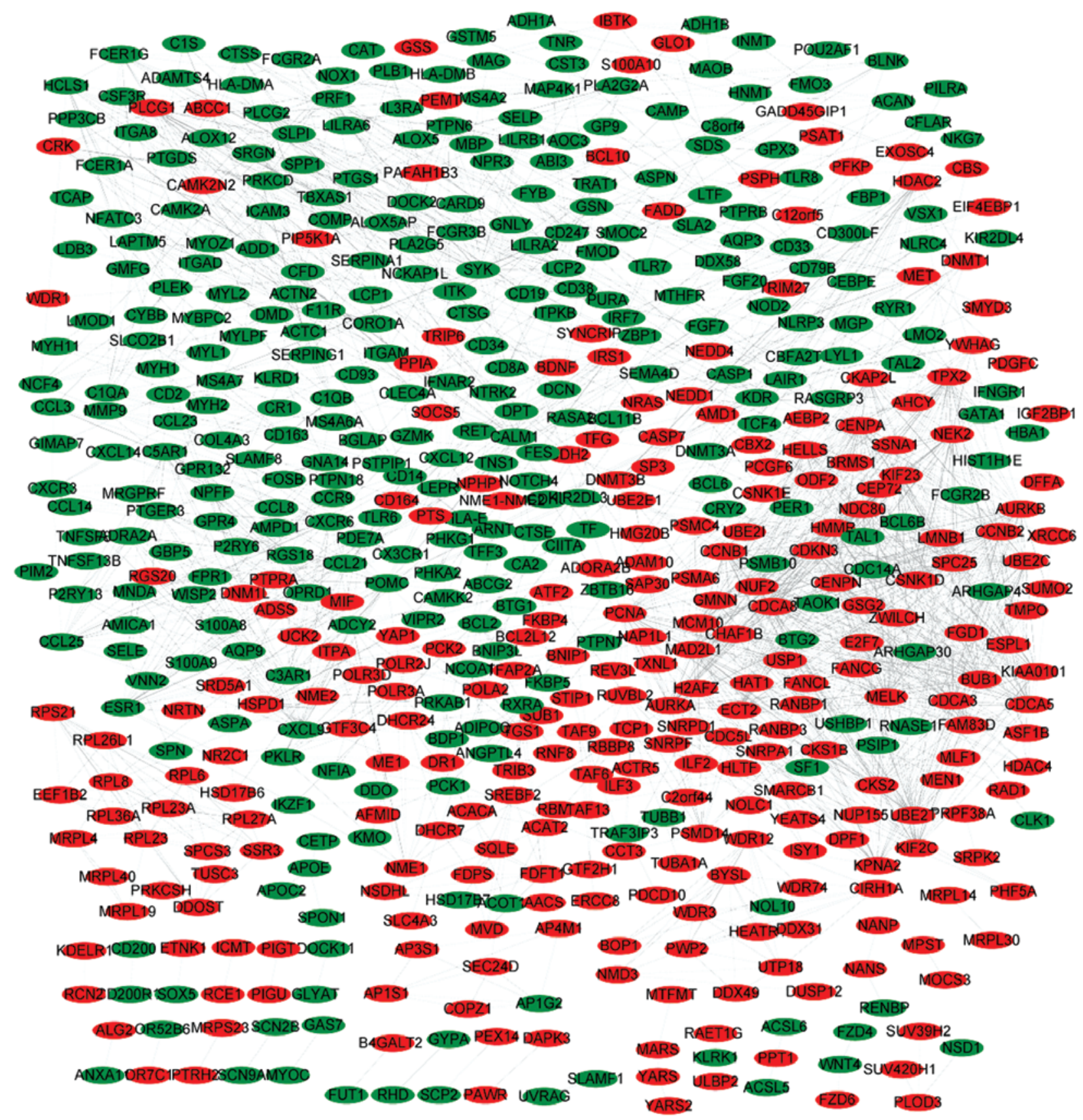

Figure 1. Protein-protein interaction network of the differentially expressed genes. The red and green circles represent for the upregulated and downregulated genes, respectively.

(C5AR1), prostaglandin E receptor 3 (PTGER3) and formyl peptide receptor 1 (FPR1).

Module F had 45 nodes and 609 interactions. In this module, the number of upregulated genes was significantly more than the number of downregulated genes. For the DEGs in module $\mathrm{F}$, the enriched KEGG pathways included oocyte meiosis $(\mathrm{P}=1.56 \mathrm{E}-05)$, cell cycle $(\mathrm{P}=2.91 \mathrm{E}-05)$ and progesterone-mediated oocyte maturation $(\mathrm{P}=0.0022)$. Notably, cyclin B1 (CCNB1; degree $=40)$, aurora kinase A (AURKA; degree=39), MAD2 mitotic arrest defective-like 1 (MAD2L1; degree $=38$ ), cell division cycle associated 8 (CDCA8; degree $=38$ ), budding uninhibited by benzimidazoles 1 (BUB1; degree=37) and maternal embryonic leucine zipper kinase (MELK; degree=37) exhibited higher degrees of connectivity. In addition, several interactive associations were identified, including MAD2L1-AURKA, MAD2L1-CDCA8, MAD2L1-BUB1 and MAD2L1-MELK.

\section{Discussion}

In the present study, a total of 1,170 DEGs were screened, including 530 upregulated genes and 640 downregulated genes. The enriched pathways for the DEGs included steroid biosynthesis and ribosome. In particular, AURKA $($ degree $=39)$, MAD2L1 (degree $=38)$, CDCA8 $($ degree $=38)$, BUB1 (degree=37) and MELK (degree=37) exhibited higher 

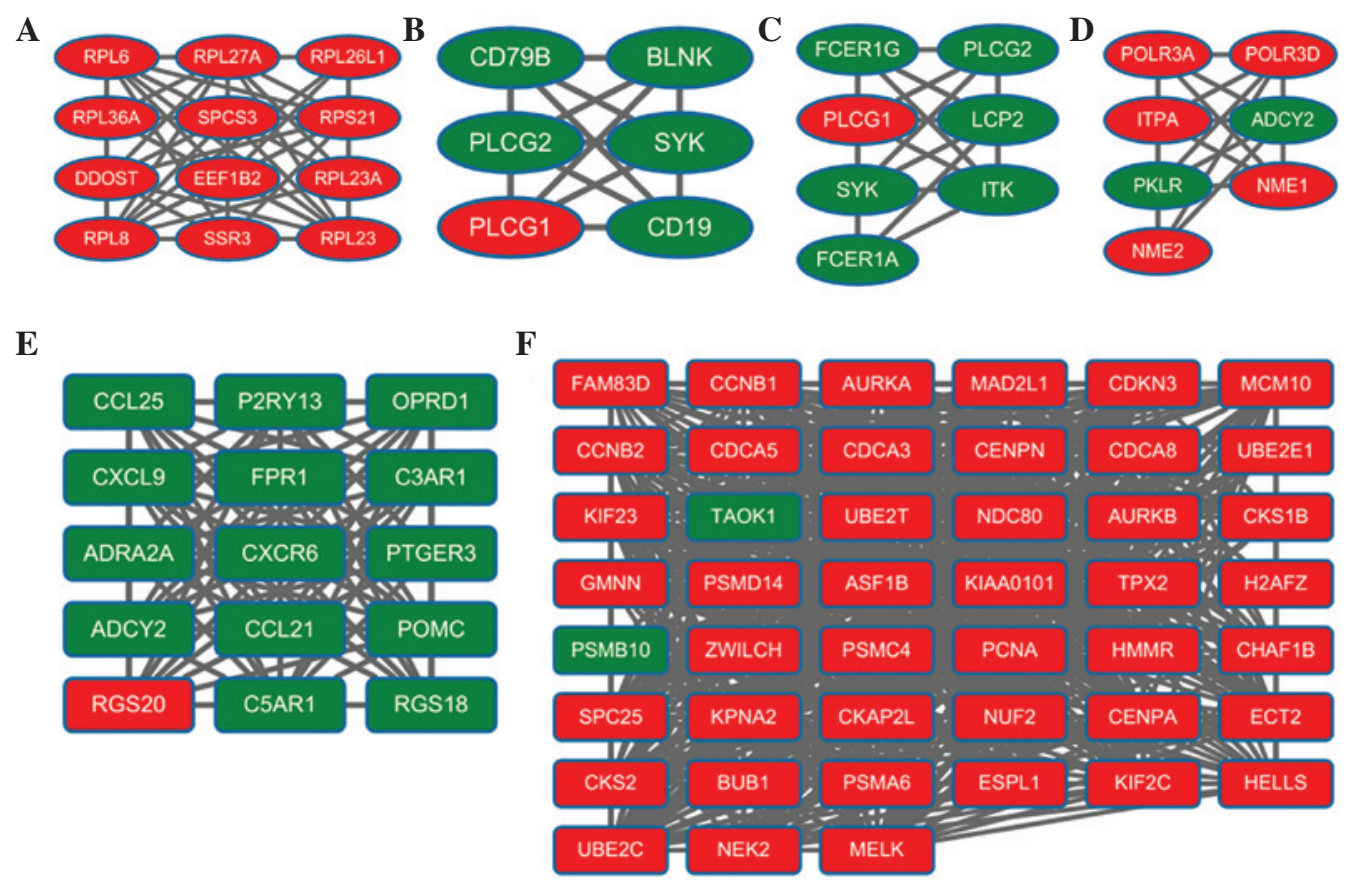

Figure 2. The top six modules (A-F) obtained from the protein-protein interaction network of the differentially expressed genes. The circular and square nodes represent proteins and the grey lines represent interactions. Red circles indicate upregulated genes and green circles represent downregulated genes.

Table I. The top 10 most enriched GO functions for the differentially expressed genes.

\begin{tabular}{|c|c|c|c|c|c|c|}
\hline Gene & Category & ID & Term & $\begin{array}{l}\text { Number } \\
\text { of genes }\end{array}$ & $\begin{array}{l}\text { Examples of } \\
\text { genes }\end{array}$ & P-value \\
\hline \multirow[t]{5}{*}{ Upregulated } & \multirow[t]{5}{*}{ BP } & GO:0048285 & Organelle fission & 56 & KIF23, CEP72 & 1.19E-09 \\
\hline & & GO:0022402 & Cell cycle process & 45 & BCAT1, KIF23 & $3.90 \mathrm{E}-09$ \\
\hline & & GO:0000280 & Nuclear division & 26 & KIF23, CEP72 & 8.03E-09 \\
\hline & & GO:0000278 & Mitotic cell cycle & 35 & KIF23, BCAT1 & 4.44E-09 \\
\hline & & GO:0007067 & Mitosis & 26 & MAD2L1, CCNB2 & $8.03 \mathrm{E}-09$ \\
\hline \multirow[t]{5}{*}{ Downregulated } & \multirow[t]{5}{*}{ BP } & GO:0006955 & Immune response & 100 & AQP9, TLR2 & $5.15 \mathrm{E}-32$ \\
\hline & & GO:0006952 & Defense response & 89 & KIR2DL3, CD300LB & $2.69 \mathrm{E}-28$ \\
\hline & & GO:0006954 & Inflammatory response & 53 & MBP, CD96 & 4.47E-19 \\
\hline & & GO:0002684 & $\begin{array}{l}\text { Positive regulation of } \\
\text { immune system process }\end{array}$ & 43 & CCL23, HRH2 & $3.47 \mathrm{E}-17$ \\
\hline & & GO:0009611 & Response to wounding & 63 & FGF7, ACVRL1 & $1.40 \mathrm{E}-15$ \\
\hline
\end{tabular}

GO, Gene Ontology; BP, biological process.

degrees of connectivity in module F of the PPI network of the DEGs.

As a member of the L2P family of ribosomal proteins, RPL8 is a component of the 60S ribosomal subunit in eucaryotic cells (23). Amplification of RPL8 may be associated with the pathogenesis of OS (24). RPL8 can respond to the chemotherapy in conventional OS, and it may be beneficial in the assessment at diagnosis and for stratifying participants of randomized trials (25). A daily-repeated ribosome biogenesis inhibition can result in progressive reduction of ribosome content and extinction of protein- and p53-deficient human OS cell lines (26). In module A, the enriched ribosome pathway involved RPL8, indicating that RPL8 may be involved in OS.
PLC is important in mediating intracellular signal transduction (27). As a member of the PLC family, PLC $\gamma 1$ is upregulated in several cancer cell lines and cancer tissues (28). It is also reported that PLC $\gamma 1$ promotes the invasion of several types of tumor (29-31). PLC $\gamma 2$ translocation is essential in transmitting TPA signal to PKC $\alpha$ and PKC $\alpha$ can directly promote the apoptosis of human cancer cell lines, thus, PLC $\gamma 2$ is critical in the process of inducting apoptosis (32). The SYK gene can be reactivated by inhibition of DNA promoter methylation in human cancer and may act as a tumor suppressor (33). The B-cell receptor signaling pathway has been correlated with OS (34). In module B in the present study, the B-cell receptor signaling pathway involved PLC $\gamma 1$, 
Table II. The top 10 most enriched Kyoto Encyclopedia of Genes and Genomes pathways for the differentially expressed genes.

\begin{tabular}{|c|c|c|c|c|}
\hline Gene & Name & $\begin{array}{l}\text { Number } \\
\text { of genes }\end{array}$ & $\begin{array}{c}\text { Examples of } \\
\text { genes }\end{array}$ & P-value \\
\hline \multirow[t]{5}{*}{ Upregulated } & hsa00100:Steroid biosynthesis & 5 & SQLE, DHCR7 & 0.0023 \\
\hline & hsa00270:Cysteine and methionine metabolism & 6 & AHCY, DNMT1 & 0.0056 \\
\hline & hsa03040:Spliceosome & 11 & SNRPA1, MAGOH & 0.0110 \\
\hline & hsa00970:Aminoacyl-tRNA biosynthesis & 6 & YARS, NARS2 & 0.0125 \\
\hline & hsa03010:Ribosome & 8 & RPL6, RPL8 & 0.0294 \\
\hline \multirow[t]{5}{*}{ Downregulated } & hsa04514:Cell adhesion molecules & 20 & HLA-DQB1, F11R & $3.74 \mathrm{E}-05$ \\
\hline & hsa05310:Asthma & 9 & CD2, SELE & $7.43 \mathrm{E}-05$ \\
\hline & hsa04640:Hematopoietic cell lineage & 15 & IL1R2, CR1 & $1.05 \mathrm{E}-04$ \\
\hline & hsa05332:Graft-versus-host disease & 10 & PRF1, KIR2DL3 & $1.21 \mathrm{E}-04$ \\
\hline & hsa04650:Natural killer cell mediated cytotoxicity & 19 & PRF1, PTPN6 & $1.36 \mathrm{E}-04$ \\
\hline
\end{tabular}

Table III. Enriched Kyoto Encyclopedia of Genes and Genomes pathways for differentially expressed genes in modules A-F.

\begin{tabular}{|c|c|c|c|c|}
\hline Module & Name & $\begin{array}{l}\text { Number } \\
\text { of genes }\end{array}$ & $\begin{array}{l}\text { Examples of } \\
\text { genes }\end{array}$ & P-value \\
\hline A & hsa03010:Ribosome & 8 & RPL6, RPL8 & $2.65 \mathrm{E}-12$ \\
\hline \multirow[t]{7}{*}{$\mathrm{B}$} & hsa04662:B cell receptor signaling pathway & 5 & PLC $\gamma 2$, SYK & $2.16 \mathrm{E}-07$ \\
\hline & hsa04664:Fc epsilon RI signaling pathway & 3 & PLC $\gamma 1$, PLC $\gamma 2$, SYK & 0.0023 \\
\hline & hsa04666:Fc gamma R-mediated phagocytosis & 3 & PLC $\gamma 1$, PLC $\gamma 2$, SYK & 0.0033 \\
\hline & hsa04650:Natural killer cell mediated cytotoxicity & 3 & PLC $\gamma 1$, PLC $\gamma 2$, SYK & 0.0064 \\
\hline & hsa05340:Primary immunodeficiency & 2 & CD19, BLNK & 0.0340 \\
\hline & hsa04664:Fc epsilon RI signaling pathway & 6 & PLC $\gamma 1$, PLC $\gamma 2$, SYK & 4.43E-09 \\
\hline & hsa04650:Natural killer cell mediated cytotoxicity & 5 & PLC $\gamma 1$, PLC $\gamma 2$, SYK & $6.45 \mathrm{E}-06$ \\
\hline \multirow[t]{4}{*}{$\mathrm{C}$} & hsa04666:Fc gamma R-mediated phagocytosis & 3 & PLC $\gamma 1$, PLC $\gamma 2$, SYK & 0.0049 \\
\hline & hsa04660:T cell receptor signaling pathway & 3 & ITK, PLC $\gamma 1$, LCP2 & 0.0063 \\
\hline & hsa04670:Leukocyte transendothelial migration & 3 & ITK, PLC $\gamma 1$, PLC $\gamma 2$ & 0.0075 \\
\hline & hsa05310:Asthma & 2 & FCER1A, FCER1G & 0.0338 \\
\hline \multirow[t]{4}{*}{$\mathrm{D}$} & hsa00230:Purine metabolism & 6 & ADCY2, NME1, PKLR & $2.31 \mathrm{E}-08$ \\
\hline & hsa00240:Pyrimidine metabolism & 5 & NME2, NME1 & $6.15 \mathrm{E}-05$ \\
\hline & hsa03020:RNA polymerase & 2 & POLR3A, POLR3D & 0.0272 \\
\hline & hsa04623:Cytosolic DNA-sensing pathway & 2 & POLR3A, POLR3D & 0.0529 \\
\hline \multirow[t]{2}{*}{$\mathrm{E}$} & hsa04080:Neuroactive ligand-receptor interaction & 7 & C5AR1, PTGER3, FPR1 & $1.10 \mathrm{E}-05$ \\
\hline & hsa04062:Chemokine signaling pathway & 5 & $\begin{array}{l}\text { CCL25, ADCY2, CCL21, } \\
\text { CXCR6, CXCL9 }\end{array}$ & $6.95 \mathrm{E}-04$ \\
\hline \multirow[t]{6}{*}{$\mathrm{F}$} & hsa04060:Cytokine-cytokine receptor interaction & 4 & CCL25, CCL21, CXCR6, CXCL9 & 0.0210 \\
\hline & hsa04114:Oocyte meiosis & 6 & & \\
\hline & hsa04110:Cell cycle & 6 & $\begin{array}{l}\text { CCNB1, CCNB2, MAD2L1, } \\
\text { BUB1, ESPL1, AURKA }\end{array}$ & \\
\hline & & & $\begin{array}{l}\text { CCNB1, CCNB2, MAD2L1, } \\
\text { BUB1, PCNA, ESPL1 }\end{array}$ & $\begin{array}{l}1.56 \mathrm{E}-05 \\
2.91 \mathrm{E}-05\end{array}$ \\
\hline & hsa03050:Proteasome & 4 & $\begin{array}{l}\text { PSMB 10, PSMD14, } \\
\text { PSMA6, PSMC4 }\end{array}$ & 3.81E-04 \\
\hline & hsa04914:Progesterone-mediated oocyte maturation & 4 & CCNB1, CCNB2, MAD2L1, BUB1 & 0.0022 \\
\hline
\end{tabular}

SYK and PLC $\gamma 2$. The Fc-epsilon RI signaling pathway involves multiple component genes that are altered at the chromosome level, and may be associated with the pathogenesis of
OS (5). In module $\mathrm{C}$, the $\mathrm{Fc}$-epsilon RI signaling pathway also involved PLC $\gamma 1$, SYK and PLC $\gamma 2$. Thus, it was hypothesized that PLC $\gamma 1$, SYK and PLC $\gamma 2$ may be closely correlated with 
OS. In addition, in modules B and C, the upregulated PLC $\gamma 1$ was observed to have an interactive association with downregulated SYK and PLC $\gamma 2$, indicating that PLC $\gamma 1$ may also be involved in OS by mediating SYK and PLC $\gamma 2$.

The overexpression of MAD2 can be caused by retinoblastoma pathway inactivation and is associated with carcinogenesis (35). The expression of MAD2 is upregulated in human OS, and its overexpression is involved in earlier metastasis and poorer survival rates in patients with human OS (36). Knockdown of MAD2 can induce OS cell apoptosis, involving the cleavage of $\operatorname{Rad} 21$ (37). This suggests that MAD2L1 may be closely correlated with OS. The mitotic checkpoint gene, BUB1, may also drive tumor metastasis and progression (38). CDCA8 and aurora kinase B (AURKB) are overexpressed in tumor cells $(39,40)$ and selective suppression of the CDCA8-AURKB pathway may be a promising therapeutic strategy in the treatment of cancer (41). MELK is a protein kinase and candidate oncoprotein, which is dysregulated in several types of cancer (42-44), as well as being involved in resistance to apoptosis (43). In module $F$ in the present study, MAD2L1 had interactive associations seperately with AURKA, CDCA8, BUB1 and MELK, suggesting that MAD2L1 may also be involved in OS by mediating AURKA, CDCA8, BUB1 and MELK.

In conclusion, the present study performed a comprehensive bioinformatics analysis of genes, which may be involved in OS. A total of 1,170 DEGs were screened, which including 530 upregulated genes and 640 downregulated genes. The results of the subsequent analyses suggested that RPL8, PLC $\gamma 1$, PLC $\gamma 2$, SYK, MAD2L1, AURKA, CDCA8, BUB1 and MELK may be correleted with OS. However, further investigations are required to elucidate their mechanisms of action in OS.

\section{References}

1. Ottaviani G and Jaffe N: The epidemiology of osteosarcoma Cancer Treat Res 152: 3-13, 2009.

2. Ritter J and Bielack SS: Osteosarcoma. Ann Oncol 21 (Suppl 7): vii320-vii325, 2010

3. Stiller CA, Bielack SS, Jundt G and Steliarova-Foucher E: Bone tumours in European children and adolescents, 1978-1997. Report from the automated childhood cancer information system project. Eur J Cancer 42: 2124-2135, 2006.

4. Stiller CA, Craft AW and Corazziari I; EUROCARE Working Group: Survival of children with bone sarcoma in Europe since 1978: results from the EUROCARE study. Eur J Cancer 37: 760-766, 2001.

5. Yang J, Yang D, Sun Y, et al: Genetic amplification of the vascular endothelial growth factor (VEGF) pathway genes, including VEGFA, in human osteosarcoma. Cancer 117: 4925-4938, 2011.

6. Quan GM and Choong PF: Anti-angiogenic therapy for osteosarcoma. Cancer Metast Rev 25: 707-713, 2006.

7. Dvorak HF: Vascular permeability factor/vascular endothelial growth factor: A critical cytokine in tumor angiogenesis and a potential target for diagnosis and therapy. J Clin Oncol 20: 4368-4380, 2002.

8. Lee Y, Tokunaga T, Oshika Y, et al: Cell-retained isoforms of vascular endothelial growth factor (VEGF) are correlated with poor prognosis in osteosarcoma. Eur J Cancer 35: 1089-1093, 1999.

9. Takenaka K, Yamagishi SI, Jinnouchi Y, Nakamura K, Matsui T and Imaizumi T: Pigment epithelium-derived factor (PEDF)-induced apoptosis and inhibition of vascular endothelial growth factor (VEGF) expression in MG63 human osteosarcoma cells. Life Sci 77: 3231-3241, 2005.

10. Miller CW, Aslo A, Tsay C, et al: Frequency and structure of p53 rearrangements in human osteosarcoma. Cancer Res 50: 7950-7954, 1990.
11. Mousses S, Mcauley L, Bell RS, Kandel R and Andrulis IL: Molecular and immunohistochemical identification of p53 alterations in bone and soft tissue sarcomas. Mod Pathol 9: 1-6, 1996.

12. Eliseev R, Dong Y, Sampson E, et al: Runx2-mediated activation of the Bax gene increases osteosarcoma cell sensitivity to apoptosis. Oncogene 27: 3605-3614, 2008.

13. Onda M, Matsuda S, Higaki S, et al: ErbB-2 expression is correlated with poor prognosis for patients with osteosarcoma. Cancer 77: 71-78, 1996.

14. Namløs HM, Meza-Zepeda LA, et al: Modulation of the osteosarcoma expression phenotype by microRNAs. PloS One 7: e48086, 2012.

15. Irizarry RA, Hobbs B, Collin F, et al: Exploration, normalization and summaries of high density oligonucleotide array probe level data. Biostatistics 4: 249-264, 2003.

16. Gentleman R, Carey VJ, Huber W, Irizarry RA and Dudoit S (eds): Bioinformatics and computational biology solutions using R and Bioconductor. Vol. 746718470. Springer, New York, NY, 2005.

17. Huang da W, Sherman BT and Lempicki RA: Systematic and integrative analysis of large gene lists using DAVID bioinformatics resources. Nat Protoc 4: 44-57, 2008.

18. Harris MA, Clark J, Ireland A, et al: The Gene Ontology (GO) database and informatics resource. Nucleic Acids Res 32: D258-D261, 2004.

19. Lu M, Zhang Q, Deng M, et al: An analysis of human microRNA and disease associations. PloS One 3: e3420, 2008.

20. Franceschini A, Szklarczyk D, Frankild S, et al: STRING v9.1: Protein-protein interaction networks, with increased coverage and integration. Nucleic Acids Res 41: D808-D815, 2013.

21. Saito R, Smoot ME, Ono K, et al: A travel guide to Cytoscape plugins. Nat Med 9: 1069-1076, 2012.

22. Palla G, Derényi I, Farkas I and Vicsek T: Uncovering the overlapping community structure of complex networks in nature and society. Nature 435: 814-818, 2005.

23. Cui XR, Hou WR, Yang J, Hou Y and Peng ZS: Cloning and sequence analysis of ribosomal protein L8 gene (RPL8) from the (Ailuropoda melanoleuca). In: 2011 4th International Conference on Biomedical Engineering and Informatics, Shanghai, China, pp1407-1411 2011.

24. Yang J and Zhang W: New molecular insights into osteosarcoma targeted therapy. Curr Opin Oncol 25: 398-406, 2013.

25. Salas S, Jézéquel P, Campion L, Deville JL, Chibon F, Bartoli C, Gentet JC, Charbonnel C, Gouraud W, Voutsinos-Porche B, et al: Molecular characterization of the response to chemotherapy in conventional osteosarcomas: Predictive value of HSD17B10 and IFITM2. Int J Cancer 125: 851-860, 2009.

26. Montanaro L, Mazzini G, Barbieri S, et al: Different effects of ribosome biogenesis inhibition on cell proliferation in retinoblastoma protein-and p53-deficient and proficient human osteosarcoma cell lines. Cell Prolif 40: 532-549, 2007.

27. Fukami K: Structure, regulation and function of phospholipase $C$ isozymes. J Biochem 131: 293-299, 2002.

28. Noh DY, Kang H, Kim YC, et al: Expression of phospholipase $\mathrm{C}$-gamma 1 and its transcriptional regulators in breast cancer tissues. Anticancer Res 18: 2643-2648, 1997.

29. Mamoune A, Kassis J, Kharait S, Kloeker S, Manos E, Jones DA and Wells A: DU145 human prostate carcinoma invasiveness is modulated by urokinase receptor (uPAR) downstream of epidermal growth factor receptor (EGFR) signaling. Exp Cell Res 299: 91-100, 2004.

30. Kochhar KS and Iyer AP: Hepatocyte growth factor induces activation of Nck and phospholipase C- $\gamma$ in lung carcinoma cells. Cancer Lett 104: 163-169, 1996.

31. Kassis J, Radinsky R and Wells A: Motility is rate-limiting for invasion of bladder carcinoma cell lines. Int J Biochem Cell Biol 34: 762-775, 2002.

32. Zhang B, Wu Q, Ye XF, Liu S, Lin XF and Chen MC: Roles of PLC-gamma2 and PKCalpha in TPA-induced apoptosis of gastric cancer cells. World J Gastroenterol 9: 2413-2418, 2003.

33. Dong S, Ma L, Xu N, et al: Research on the reactivation of Syk expression caused by the inhibition of DNA promoter methylation in the lung cancer. Neoplasma 58: 89-95, 2010.

34. Luo P, Yang X, Ying M, et al: Retinoid-suppressed phosphorylation of RAR $\alpha$ mediates the differentiation pathway of osteosarcoma cells. Oncogene 29: 2772-2783, 2010.

35. Agoston AT, Argani P, De Marzo AM, Hicks JL and Nelson WG: Retinoblastoma pathway dysregulation causes DNA methyltransferase 1 overexpression in cancer via MAD2-mediated inhibition of the anaphase-promoting complex. Am J Pathol 170: 1585-1593, 2007. 
36. Yu L, Guo WC, Zhao SH, Tang J and Chen JL: Mitotic arrest defective protein 2 expression abnormality and its clinicopathologic significance in human osteosarcoma. Apmis 118: 222-229, 2010

37. Yu L, Guo W, Zhao S, Tang J and Liu J: Knockdown of Mad2 induces osteosarcoma cell apoptosis-involved $\operatorname{Rad} 21$ cleavage. J Orthop Sci 16: 814-820, 2011.

38. Shichiri M, Yoshinaga K, Hisatomi H, Sugihara K and Hirata Y: Genetic and epigenetic inactivation of mitotic checkpoint genes hBUB1 and hBUBR1 and their relationship to survival. Cancer Res 62: 13-17, 2002.

39. Bischoff JR, Anderson L, Zhu Y, et al: A homologue of Drosophila aurora kinase is oncogenic and amplified in human colorectal cancers. EMBO J 17: 3052-3065, 1998.

40. Branca M, Giorgi C, Santini D, et al: Survivin as a marker of cervical intraepithelial neoplasia and high-risk human papillomavirus and a predictor of virus clearance and prognosis in cervical cancer. Am J Clin Pathol 124: 113-121, 2005.
41. Hayama S, Daigo Y, Yamabuki T, et al: Phosphorylation and activation of cell division cycle associated 8 by aurora kinase $\mathrm{B}$ plays a significant role in human lung carcinogenesis. Cancer Res 67: 4113-4122, 2007.

42. Pickard MR, Green AR, Ellis IO, et al: Dysregulated expression of Fau and MELK is associated with poor prognosis in breast cancer. Breast Cancer Res 11: R60, 2009.

43. Lin ML, Park JH, Nishidate T, Nakamura Y and Katagiri T: Involvement of maternal embryonic leucine zipper kinase (MELK) in mammary carcinogenesis through interaction with Bcl-G, a pro-apoptotic member of the Bcl-2 family. Breast Cancer Res 9: R17, 2007.

44. Gray D, Jubb AM, Hogue D, et al: Maternal embryonic leucine zipper kinase/murine protein serine-threonine kinase 38 is a promising therapeutic target for multiple cancers. Cancer Res 65: 9751-9761, 2005 